\title{
The Case of a Pregnant Woman with ARDS Due to COVID-19 Treated with Hydroxychloroquine, Azithromycin, and Remdesivir and Delivery of a Healthy Baby During Mechanical Ventilation through Cesarean Section
}

\author{
Adria Madera-Acosta ${ }^{1 *}$; Bassman Tappuni ${ }^{1}$; Monika Sadlak ${ }^{1}$; Ravleen Grewall ${ }^{\text {; } \text { Martin Gnoni }^{2} \text {; Angel Mena }}{ }^{3}$; Yousef Ali-Ahwel ${ }^{4}$ \\ ${ }^{1}$ Internal Medicine Residency, Good Samaritan Hospital, Cincinnati, OH, USA; ${ }^{2}$ University of Louisville, Louisville, KY, USA; ${ }^{3}$ nternal Medicine Program, Tri-Health
} Good Samaritan Hospital, Cincinnati, OH, USA; ${ }^{4}$ Critical Care, Good Samaritan Hospital, Cincinnati, OH, USA

*adria_madera-acosta@trihealth.edu

Recommended Citation: Madera-Acosta A, Tappuni B, Sadlak M, et al. The case of a pregnant woman with ARDS due to COVID-19 treated with hydroxychloroquine, azithromycin, and remdesivir and delivery of a healthy baby during mechanical ventilation through cesarean section. Univ Louisville J Respir Infect 2020; 4(1):Article 73. doi: 10.18297/ji//vol4/iss1/73.

\begin{abstract}
We are in the midst of a pandemic due to SARS-CoV-2. Pregnancy was identified among the risk factors for worse clinical outcomes in multiple studies. The optimal therapy in this group of patients remains to be defined. Here, we present the case of a 39-year-old Caucasian pregnant female at 31 weeks of gestation, who was treated successfully with hydroxychloroquine, azithromycin, remdesivir, prone therapy, and cesarean delivery of a healthy baby while on mechanical ven-
\end{abstract}

\section{Introduction}

The world as we know it has changed with the SARSCoV-2 pandemic. The virus known to cause COVID-19 and has been particularly virulent, leading to a global health crisis. COVID-19 primarily presents with respiratory compromise, often leading to acute respiratory distress syndrome (ARDS) and multi-organ failure. Multiple studies have postulated the role of thromboembolic events as a vital trigger for COVID-19 induced coagulopathy.[1] The pathogenesis of the disease remains to be determined, however observational studies suggest the integral role of endothelial activation and microthrombosis with disseminated organ damage as the cause for increased morbidity and mortality.[2] Some patient populations are noted to have a higher predisposition to COVID-19 such as those with history of asthma, chronic obstructive pulmonary disease, cardiovascular disease and pregnancy. Of note, pregnancy itself is described as a pro-coagulant state. The progression and severity of COVID-19 is not yet well defined or studied in the pregnant population. tilation. She remained SARS-CoV-2-positive until the 39th day of hospitalization. We discuss the pathophysiology of the increased risk of infections during pregnancy and particularly the high risk of microthombosis and coagulopathy due to COVID-19. Ultimately, the contribution of the medications used to the favorable outcomes remains unknown; it is more likely that the delivery helped to resolve the infection.

In this article, we describe a case of a pregnant patient who developed ARDS with accelerated respiratory failure requiring intubation and further intensive care unit (ICU) management. We hypothesize her disease process was accelerated by her pregnancy and outline her case and management here. Additionally, we performed a literature review of available treatments and compare those with the management strategies utilized in this patient

\section{Case Description}

The patient was a 39-year-old Caucasian female with a past medical history of bicuspid aortic valve and type 1 myotonic dystrophy. She presented to the Emergency Department (ED) at 31 weeks gestation with a two-day history of cough, headache and fever. She had known exposure to a COVID-19 positive individual prior to presentation. The patient was discharged home due to her mild symptoms and clinical stability. She was advised to return if symptoms worsened. 


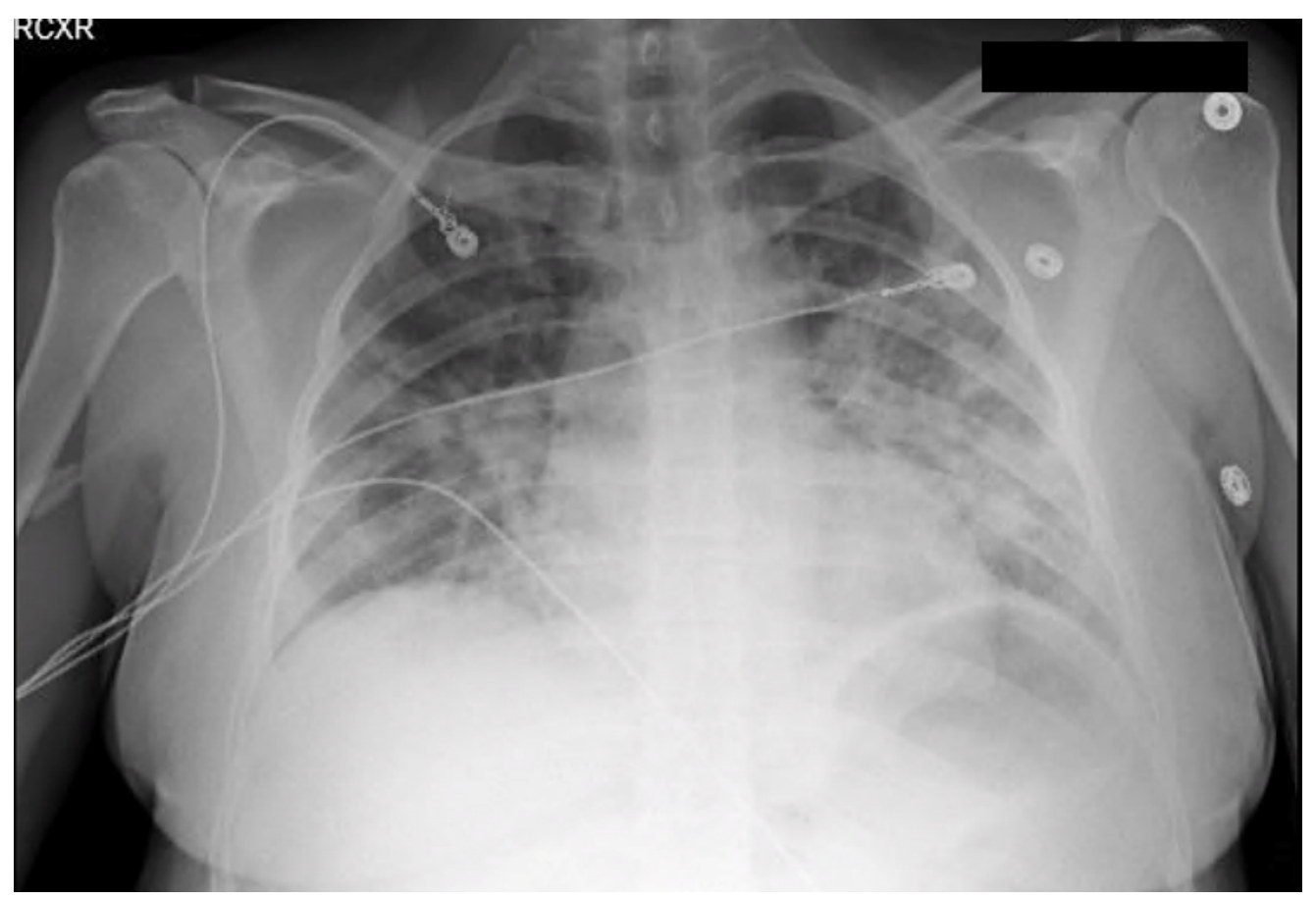

Figure 1. Patchy areas of opacity seen within both lungs, left greater than right. Infection, including both viral and bacterial pneumonia is favored over edema.

Four days later, the patient presented with worsening shortness of breath and her vitals on presentation were notable for a fever of $101.6 \mathrm{~F}$ with an oxygen saturation of $89 \%$ on room air. Chest X-ray and computed tomography angiography of the chest showed bilateral patchy infiltrates (Figure 1 and Figure 2). White blood cell count was consistent with lymphopenia and a procalcitonin was elevated at 0.33 . The patient was initially admitted to the medical floor on 3L of oxygen via nasal cannula. However, she decompensated quickly with an arterial blood gas remarkable for a $\mathrm{PaO}_{2} / \mathrm{FiO}_{2}$ ratio of 142 , consistent with moderately severe ARDS. As a result, she was transferred ICU, intubated and mechanically ventilated. The patient was started on ceftriaxone, azithromycin and hydroxychloroquine. COVID-19 infection was confirmed via nasopharyngeal swab four days after admission.

The patient's respiratory status continued to decline and proning was indicated for improved alveolar recruitment. There was hesitation to prone due to the need for continuous fetal monitoring however the anticipated benefit of proning was determined to outweigh the need for supine positioning to accommodate fetal monitoring. Despite proning, the patient developed ventilator desynchrony which required heavy sedation and cistacuronium for seven days. Ultimately a 10-day treatment course with remdesivir was initiated as the patient failed to improve with the aforementioned management.
On the ninth hospital day, the patient underwent cesarean section with safe delivery of a healthy baby boy which led to significant improvement in her ability to oxygenate. The amniotic fluid and placenta tissue culture were negative for COVID-19 infection.

The patient continued to have intermittent febrile episodes and elevation in procalcitonin which led to concern for ventilator associated pneumonia with sputum cultures growing methicillin-susceptible Staphylococcus aureus. She completed a seven-day course of cefepime and vancomycin with repeat chest X-ray indicating improvement of bilateral pulmonary infiltrates.

As she was making significant improvement in her respiratory status, the patient was extubated, however she required subsequent re-intubation within a few hours due to increased work of breathing. The patient underwent tracheostomy placement due to difficulty in weaning off mechanical ventilation. This was attributed to the combination of the patient's history of myotonic dystrophy, prolonged immobility and presumed critical care neuromuscular weakness. She made significant improvement while working with physical and occupational therapy in regaining her strength and was slowly weaned off mechanical ventilation. Repeat imaging showed continued improvement of her multifocal pneumonia. Shortly thereafter, she was transitioned to a tracheostomy collar and decannulated prior to discharge. Of note, she continued 


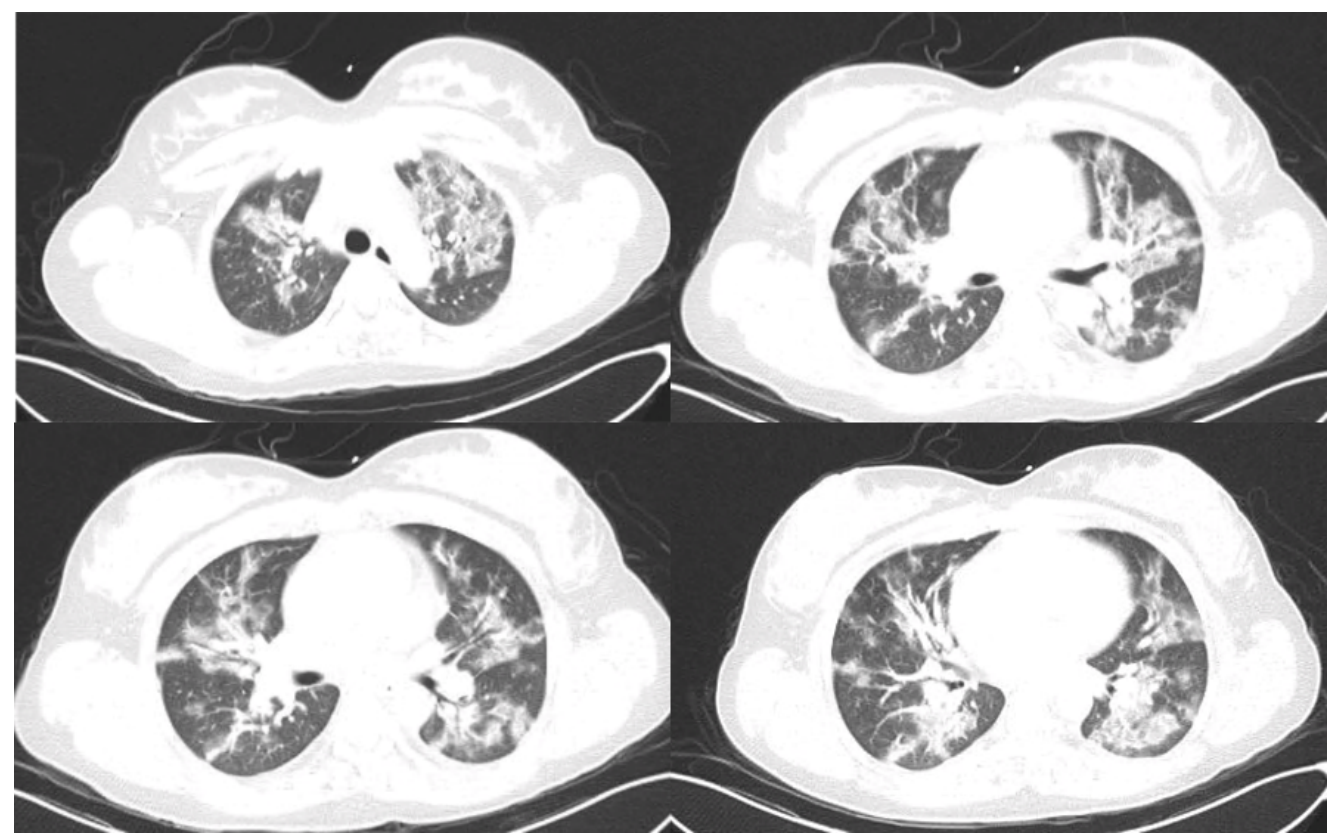

Figure 2. Heterogenous areas of patchy ground glass opacity and septal thickening bilateral most compatible with an infectious etiology.

to test positive for COVID-19 from the SARS-CoV-2 NP PCR until the 39th day of her hospital stay. The patient was able to recover from her illness after 41 days in the ICU setting and was discharged home in stable condition.

\section{Discussion}

This case highlights the rapid progression of COVID-19 infection to ARDS in vulnerable populations. Pregnant women are known to be at a higher risk of severe illness and higher mortality from viral infections noticeably during pandemics such as Ebola and influenza.[35] Infected women also have an increased incidence of cesarean delivery and preterm birth. There have been no reported cases of vertical transmission of COVID19.[6] Being a relatively new disease process, limited data is available on COVID-19 in pregnant patients. A comprehensive systematic review published recently reviewed perinatal outcomes in pregnant patients with COVID-19. A total of 114 pregnant women were included in the review and the most commonly reported symptoms were fever $(87.5 \%)$ and cough $(53.8 \%)$, followed closely by fatigue (22.5\%), myalgia (16.3\%) and dyspnea $(11.3 \%)$. The majority of studied women had a cesarean section for various reasons; however, the fetal outcomes were fairly good. Preterm birth (21.3\%) and fetal distress $(10.7 \%)$ were the most prevalent outcomes. There were no reports of neonatal infection noted, however the majority of patients were in the third trimester of pregnancy and further studies are needed to evaluate neonatal transmission of COVID-
19.[7] COVID-19 was not detected in the neonate, placenta or amniotic fluid in our case.

Pregnancy is a unique immunologic state in which the body has to adapt to the fetus-especially acting as a foreign body-with a resulting increased susceptibility to a variety of infections. The progressive uterine distention during pregnancy is the major cause for chest wall and lung volume changes. Pregnancyrelated reduction in functional residual capacity combined with increase in oxygen consumption lowers the oxygen reserve of the mother.[8] This could explain the significant improvement in oxygenation in our case after undergoing cesarean delivery. There is also evidence showing improved outcomes using higher positive end-expiratory pressure and manual prone positioning, which were utilized in our patient.[9]

The immune system plays an important role in regulation of normal and pathologic states of pregnancy. Some pro-inflammatory cytokines, namely TNF-a, IL$1 \mathrm{~b}$ and IL-6, IL-10 and regulatory T cells (T reg), have recently been found to have immunosuppressive properties in the setting of pregnancy, appearing to play a critical role in both immune stimulatory and counter regulatory functions. It has been shown in COVID19 infections that IL-6,IL-10, and TNF-a increase the expression of programmed cell death marker-1 (PD-1) causing cell death and severe disease, which eventually can be used as prognostic factors in stratification of disease severity and possibly change management.[10] In our case, the use of two immunomodulator agents (hydroxychloroquine and azithromycin) may have ex- 
acerbated the immunosuppressive state and promoted the persistence of viral carriage for over a month which could have been detrimental. It is unknown if remdesivir had any impact on this patient's clinical outcome. The delivery of the baby appears to have been the main contributing factor to improvement in oxygenation.

Severe COVID-19 has been associated with a coagulopathic state with elevated D-Dimer and fibrinogen levels.[11] Pregnancy-related hypercoagulability is an adaptive mechanism to prevent post-partum hemorrhage. Fibrinogen and thrombin levels are noted to increase up to three times in pregnancy, while protein S, an anticoagulant factor, decreases. Prolonged bed rest during pregnancy adds to the venous stasis and hypercoagulable state in pregnancy.[12] The hypercoagulable state of pregnancy plus the pro-thrombotic state of COVID-19 due to endothelial damage may explain some of the worse outcomes during pregnancy.

Specific treatment protocols for COVID-19 in pregnancy with the administration of hydroxychloroquine, azithromycin have shown that there is no significant increased risk for malformations, intrauterine death, preterm delivery or low birth weight. There is no sub-

Received: June 5, 2020

Accepted: October 29, 2020

Published: December 15, 2020

Copyright: (c) 2022 The author(s). This original article is brought to you for free and open access by ThinkIR: The University of Louisville's Institutional Repository. For more information, please contact thinkir@louisville.edu. This article is stantial evidence regarding remdesivir in pregnancy, however in this particular case, these treatments were administered in hope of changing the patient's outcome and possibly shorten disease. It is difficult to determine whether this disease responded to these medications as our patient continued to test positive for over four weeks while in the ICU or was this a natural course of the disease itself.

In conclusion, our patient's clinical course was likely prolonged due to complications from her type A blood, type 1 muscular dystrophy, worsening immunosuppression due to immunomodulatory agents, and pregnancy. Conversely, favorable factors included supportive critical care, timely cesarean delivery which improved her respiratory function and thromboprophylaxis. It is still unclear if any of the medical treatments used had any benefit as further larger studies are needed to establish this definitively. It is also unclear if any of the medical treatments had any effect on viral shedding as the patient continued to test positive for four weeks following initial testing. Of the positive contributing factors, it was felt that supportive care had the most significant impact on the patient's eventual positive outcome.

\section{References}

1. Di Renzo GC, Giardina I. Coronavirus disease 2019 in pregnancy: Consider thromboembolic disorders and thromboprophylaxis. Am J Obstet Gynecol 2020; 223(1):135. doi: 10.1016/j.ajog.2020.04.017. PMID: 32333857.

2. Bikdeli B, Madhavan MV, Jimenez D, et al. COVID-19 and thrombotic or thromboembolic disease: Implications for prevention, antithrombotic therapy, and follow-up: JACC state-ofthe-art review. J Am Coll Cardiol 2020; 75(23):2950-73. doi: 10.1016/j.jacc.2020.04.031. PMID: 32311448.

3. Kwon JY, Romero R, Mor G. New insights into the relationship between viral infection and pregnancy complications. Am J Reprod Immunol 2014; 71(5):387-90. doi: 10.1111/aji.12243. PMID: 24702790.

4. Laake I, Tunheim G, Robertson $\mathrm{AH}$, et al. Risk of pregnancy complications and adverse birth outcomes after maternal $A(\mathrm{H} 1 \mathrm{~N} 1)$ pdm09 influenza: A Norwegian populationbased cohort study. BMC Infect Dis 2018; 18(1):525. doi: 10.1186/s12879-018-3435-8. PMID: 30348103. distributed under the terms of the Creative Commons Attribution 4.0 International License (CC BY 4.0), which permits unrestricted use, distribution, and reproduction in any medium, provided the original author and source are credited.

Funding Source: The author(s) received no specific funding for this work.

Conflict of Interest: All authors declared no conflict of interest in relation to the main objective of this work.
5. Di Mascio D, Khalil A, Saccone G, et al. Outcome of coronavirus spectrum infections (SARS, MERS, COVID19) during pregnancy: A systematic review and metaanalysis. Am J Obstet Gynecol MFM 2020; 2(2):100107. doi: 10.1016/j.ajogmf.2020.100107. PMID: 32292902.

6. Karimi-Zarchi $M$, Neamatzadeh $H$, Dastgheib SA, et al. Vertical transmission of coronavirus disease 19 (COVID19) from infected pregnant mothers to neonates: A review. Fetal Pediatr Pathol 2020; 39(3):246-50. doi: 10.1080/15513815.2020.1747120. PMID: 32238084.

7. Yang Z, Wang $M$, Zhu Z, Liu Y. Coronavirus disease 2019 (COVID-19) and pregnancy: A systematic review. J Matern Fetal Neonatal Med 2020:1-4. doi: 10.1080/14767058.2020.1759541. PMID: 32354293.

8. LoMauro A, Aliverti A. Respiratory physiology of pregnancy: Physiology masterclass. Breathe (Sheff) 2015; 11(4):297-301. doi: 10.1183/20734735.008615. PMID: 27066123. 
9. Tolcher MC, McKinney JR, Eppes CS, et al. Prone positioning for pregnant women with hypoxemia due to coronavirus disease 2019 (COVID-19). Obstet Gynecol 2020; 136(2):259-61. doi: 10.1097/aog.0000000000004012. PMID: 32516274.

10. Denney JM, Nelson EL, Wadhwa PD, et al. Longitudinal modulation of immune system cytokine profile during pregnancy. Cytokine 2011; 53(2):170-7. doi: 10.1016/j.cyto.2010.11.005. PMID: 21123081.
11. Spiezia L, Boscolo A, Poletto F, et al. COVID-19-related severe hypercoagulability in patients admitted to intensive care unit for acute respiratory failure. Thromb Haemost 2020; 120(6):998-1000. doi: 10.1055/s-0040-1710018. PMID: 32316063.

12. Maiello $M$, Torella $M$, Caserta $L$, et al.[Hypercoagulability during pregnancy: Evidences for a thrombophilic state]. Minerva Ginecol 2006; 58(5):417-22. PMID: 17006429. 